OPEN ACCESS

Edited by:

Peter Sörös,

University of Oldenburg, Germany

Reviewed by:

Filippo Brighina,

Università degli Studi di Palermo, Italy

Patrizia Silvia Bisiacchi,

Università degli Studi di Padova, Italy

*Correspondence:

Leila Rahnama

I.rahnama@uswr.ac.ir

Received: 23 March 2018 Accepted: 09 October 2018

Published: 31 October 2018

Citation:

Achacheluee ST, Rahnama L, Karimi N, Abdollahi I, Arslan SA and

Jaberzadeh S (2018) The Effect

of Unihemispheric Concurrent Dual-Site Transcranial Direct Current

Stimulation of Primary Motor and Dorsolateral Prefrontal Cortices on Motor Function in Patients With

Sub-Acute Stroke.

Front. Hum. Neurosci. 12:441. doi: 10.3389/fnhum.2018.00441

\section{The Effect of Unihemispheric} Concurrent Dual-Site Transcranial Direct Current Stimulation of Primary Motor and Dorsolateral Prefrontal Cortices on Motor Function in Patients With Sub-Acute Stroke

\author{
Sahar Toluee Achacheluee ${ }^{1}$, Leila Rahnama ${ }^{1,2 *}$, Noureddin Karimi', Iraj Abdollahi', \\ Syed Asadullah Arslan ${ }^{3}$ and Shapour Jaberzadeh ${ }^{4}$ \\ ${ }^{1}$ Department of Physiotherapy, University of Social Welfare and Rehabilitation Sciences, Tehran, Iran, ${ }^{2}$ Pediatric \\ Neurorehabilitation Research Center, University of Social Welfare and Rehabilitation Sciences, Tehran, Iran, ${ }^{3}$ Department of \\ Physiotherapy, School of Rehabilitation, International Campus, Tehran University of Medical Sciences, Tehran, Iran, \\ ${ }^{4}$ Non-invasive Brain Stimulation and Neuroplasticity Laboratory, Department of Physiotherapy, School of Primary and Allied \\ Health Care, Faculty of Medicine, Monash University, Melbourne, VIC, Australia
}

It is believed that unihemispheric concurrent dual-site transcranial direct current stimulation (tDCS $U H C D S$ ) of the primary motor cortex (M1) and the dorsolateral prefrontal cortex (DLPFC) causes an increase in motor cortex excitability. However, the clinical effect of this type of stimulation on patients with neurological conditions is not yet known. The aim of the present study was to assess the effect of anodal-tDCSUHCDS (atDCS HHCDS $_{\text {) }}$ on upper limb motor function in subacute stroke patients. Fifteen patients participated in this sham-controlled crossover study. The main outcome measures were the reaction time $(R T)$ to visual stimuli, completion time of a nine-pin pegboard (9PPB), and the scores from the Fugl-Meyer assessment (FMA) for the upper limb of the involved side before and after three brain stimulation conditions. For a-tDCS $\cup H C D S$, the anodal electrodes were placed on the M1 and the DLPFC, while for a-tDCS, the anodal electrode was placed on the M1. For the sham stimulation, the tDCS was turned off after $30 \mathrm{~s}$. For brain stimulation, the selected current was $1 \mathrm{~mA}$ for 20 min. After a-tDCS $U H C D S$, there was a significant reduction in the RT and completion time of the 9-PPB compared with the times after a-tDCS and the sham stimulation: $p=0.013$ and $p=0.022$, respectively). However, there was no significant difference in the FMA scores after the three types of stimulations $(p=0.085)$. Compared with a-tDCS, a-tDCS UHCDS temporarily improved the RT and dexterity of the involved hand in subacute stroke patients.

Clinical Trial Registration: Iranian Registry of Clinical Trials (IRCT), identifier IRCT2015012520787N1.

Keywords: tDCS, stroke rehabilitation, upper extremity, motor skills, motor cortex, stroke 


\section{INTRODUCTION}

Stroke is considered to be the second-leading cause of mortality and the major reason for disability in adults all over the world (Kuklina et al., 2012). Stroke causes motor, sensory, and cognitive deficits (Mayo et al., 1999). Upper limb weakness, which is seen in more than $40 \%$ of stroke patients, is one of the most important symptoms in both acute and chronic conditions (Parker et al., 1986). After a stroke, the recovery of motor function is very important because it enables stroke survivors to independently perform activities of daily living (Harris and Eng, 2007). Recently, a number of studies have investigated the impact of non-invasive brain stimulation (NIBS) on the enhancement of neuroplasticity and the recovery of symptoms caused by brain lesions (Hummel and Cohen, 2006; Summers et al., 2007; Kim et al., 2016; Satow et al., 2016; Andrade et al., 2017). Transcranial direct current stimulation (tDCS) is a NIBS technique that uses low-intensity direct current to modulate the excitability of neurons in different cortical sections and deep areas of the brain (Nitsche and Paulus, 2000; Liebetanz et al., 2002; Kim et al., 2012). Previous studies have suggested that the application of tDCS to the primary motor cortex (M1) can improve upper limb motor function. For example, anodal tDCS (a-tDCS) of the M1 improved the motor function of the hand in both healthy individuals and stroke patients (Bastani and Jaberzadeh, 2012; Butler et al., 2013; Lee and Lee, 2015; Fleming et al., 2017). A-tDCS may also lead to a decrease in reaction time (RT) in patients performing different motor tasks (Hummel and Cohen, 2005; Hummel et al., 2005, 2006; Boggio et al., 2006). It has been observed that separate a-tDCS of the M1 and the dorsolateral prefrontal cortex (DLPFC) increases the excitability of the motor cortex in healthy individuals (Vaseghi et al., 2015a). In addition, the simultaneous stimulation of the M1 of both hemispheres (anodal current over M1 and cathodal current over the contralateral M1) had a greater improvement on motor learning compared with a sham simulation or a unilateral a-tDCS of the M1 (Karok and Witney, 2013; Di Lazzaro et al., 2014). Stagg et al. (2013) reported that a-tDCS of the DLPFC increased the blood flow between the sensorimotor cortex and the DLPFC. Vaseghi et al. (2015b) compared a single-site stimulation of the M1 or DLPFC with the simultaneous unilateral stimulation of M1 and DLPFC using a new protocol called unihemispheric concurrent dual-site a-tDCS (a-tDCS $\left.{ }_{U H C D S}\right)$; they found that a-tDCS ${ }_{U H C D S}$ significantly increases the M1 corticospinal excitability (CSE) in healthy individuals. However, in spite of the significance of this finding in the enhancement of CSE, the clinical and functional implications of a-tDCS ${ }_{U H C D S}$ have not yet been studied. To the best of the authors' knowledge, a study of the effects of this novel tDCS approach on the motor function of stroke patients has not been conducted.

In the current research, we aimed to compare the effects of conventional single-site stimulation of M1 with a-tDCS ${ }_{U H C D S}$ on upper limb motor function. We hypothesized that a-tDCS ${ }_{U H C D S}$ of the M1-DLPFC decreases the RT and completion time for a nine-pin pegboard (9-PPB) and increases motor function as evidenced by the Fugl-Meyer assessment (FMA) in subacute stroke patients.

\section{MATERIALS AND METHODS}

\section{Design}

This study had a sham-controlled crossover design. The current study was registered as a clinical trial study in the Iranian Registry of Clinical Trials ${ }^{1}$ with the registration number: IRCT2015012520787N1. All experimental procedures were approved by the Human Ethics Committee of the University of Social Welfare and Rehabilitation Science, Tehran, Iran. This study followed the CONSORT checklist, which is included as a Supplementary File. All participants read and signed a written informed consent form before taking part in the study.

\section{Participants}

Fifteen subacute stroke patients with subcortical lesions voluntarily participated in this study; there were six females and nine males with the average age of $66.17 \pm 6.36$ years and $63.33 \pm 7.14$ years, respectively. The participants were selected from a pool of patients undergoing rehabilitation at regional clinics and hospitals. All participants sustained ischemic stroke diagnosed by a neurologist using magnetic resonance imaging. Patients were included in the study if it was their first stroke, they were $40-80$ years old, and they had a Brunnstrom score of 3 (marked spasticity, but voluntary synergistic finger movement could be observed). Patients were excluded from the study if they had any other neurological disease except stroke, they had a metal implant in the brain, they had musculoskeletal disorders that affected the upper limbs, they had aphasia, they were taking neuropsychiatric drugs, such as benzodiazepines or antidepressants, and they had scored less than 25 in the Mini-Mental State Examination (MMSE). Table 1 shows the demographic characteristics of the patients and Figure 1 shows the trial procedure flow.

\section{Transcranial Direct Current Stimulation}

Three experimental conditions (stimulation type) included a-tDCS ${ }_{U H C D S}$, conventional single-site a-tDCS, and sham a-tDCS. Each participant randomly received all three types of stimulation. A-tDCS ${ }_{U H C D S}$ was applied using two active electrodes $(2 \mathrm{~cm} \times 3 \mathrm{~cm})$ placed over the M1 and DLPFC of involved hemisphere and two reference electrodes $(2 \mathrm{~cm} \times 6 \mathrm{~cm})$ placed over the contralateral supraorbital area. For the conventional single-site a-tDCS, one active electrode $(2 \mathrm{~cm} \times 3 \mathrm{~cm})$ was located over M1 of involved hemisphere and a reference electrode $(2 \mathrm{~cm} \times 6 \mathrm{~cm})$ was placed over the contralateral supraorbital area. For the sham stimulation, the electrodes were placed in the same positions used for the stimulation of M1 or M1-DLPFC, but the device was turned off after $30 \mathrm{~s}$ of stimulation. The a-tDCS devices were set to deliver $1 \mathrm{~mA}$ direct current for $20 \mathrm{~min}$. The current density under the active electrode was $0.016 \mathrm{~mA} / \mathrm{cm}^{2}$, which was comparable with the current density used in previous studies (Furubayashi et al., 2008; Kwon et al., 2008). Small active electrodes were used in all conditions 
TABLE 1 | Patients demographic characteristics.

\begin{tabular}{|c|c|c|c|c|c|c|}
\hline Patient no. & Age (year) & Time aafter stroke (month) & Lesion site (ischemic site) & Dominant hand & MMSE & Brunnstrom \\
\hline 2 & 53 & 8 & L pontine & $\mathrm{R}$ & 27 & 4 \\
\hline 4 & 62 & 9 & L putamen thalamus & $\mathrm{R}$ & 26 & 3 \\
\hline 5 & 74 & 20 & R internal capsule & $\mathrm{R}$ & 28 & 3 \\
\hline 6 & 69 & 12 & L putamen & L & 28 & 3 \\
\hline 8 & 55 & 16 & $\mathrm{R}$ corona radiata & $\mathrm{R}$ & 27 & 4 \\
\hline 9 & 63 & 24 & $\mathrm{R}$ corona radiata & $\mathrm{R}$ & 28 & 4 \\
\hline 10 & 61 & 19 & L basal ganglia & L & 25 & 4 \\
\hline 11 & 62 & 20 & L putamen & L & 29 & 4 \\
\hline 12 & 70 & 22 & R internal capsule & $\mathrm{R}$ & 25 & 3 \\
\hline 13 & 60 & 17 & $\mathrm{R}$ basal ganglia & $\mathrm{R}$ & 29 & 4 \\
\hline
\end{tabular}

$R$, right; $L$, left.

to increase the focality of the stimulation over the target areas (Nitsche et al., 2007; Kwon et al., 2008; Bastani and Jaberzadeh, 2013). The stimulation sites were based on the international 10-20 electroencephalography standard (Figure 2). Depending on the side of the pathology, active electrodes were located over the right or left M1 (C3 or C4) and right or left DLPFC (F3 or F4). The wash-out period between the different experimental conditions was $72 \mathrm{~h}$ (Fujimoto et al., 2016).

\section{Outcome Measures}

\section{Reaction Time}

Deary-Liewald reaction time software (RT, version 3.10, Centre for Cognitive Ageing and Cognitive Epidemiology, University

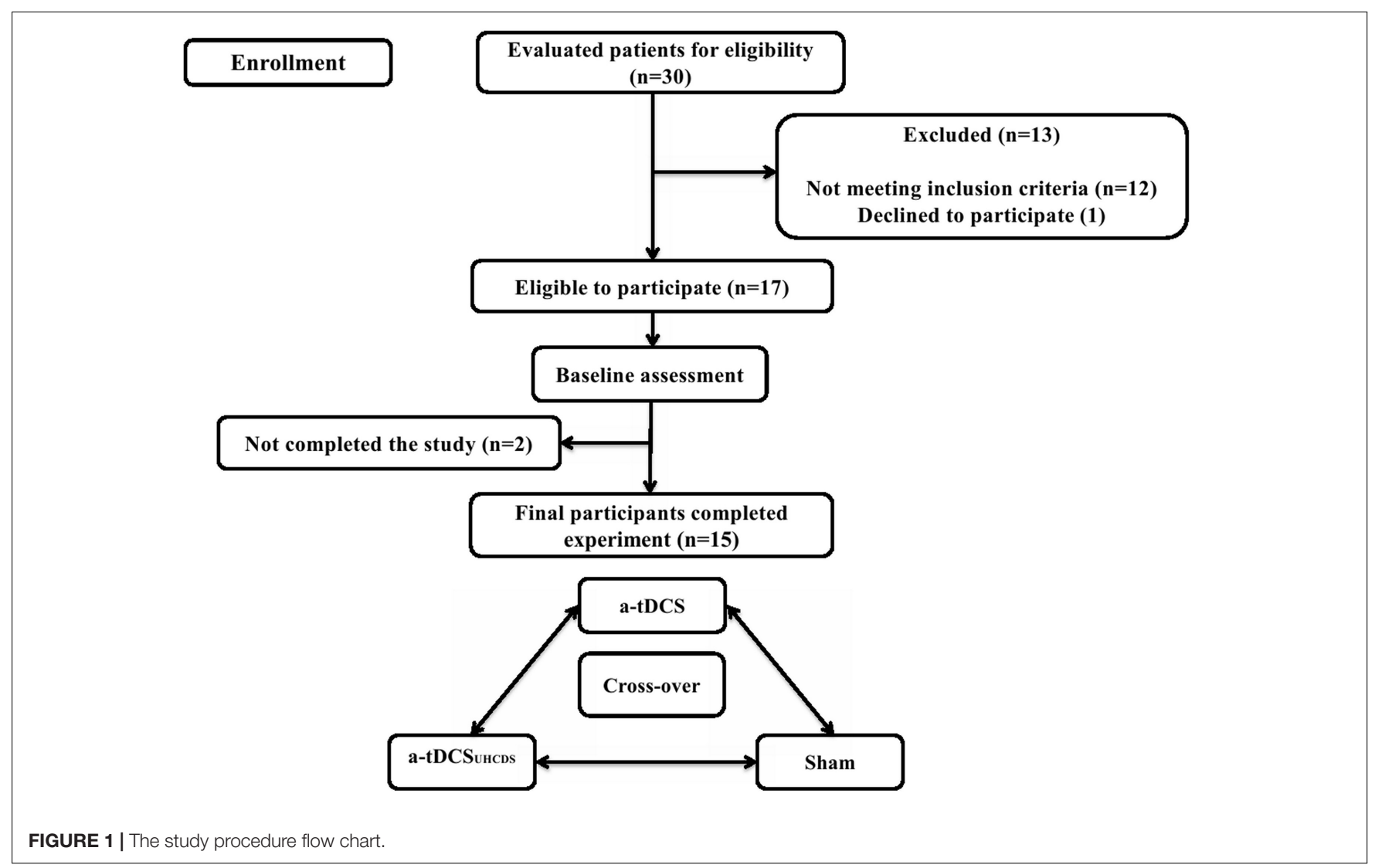



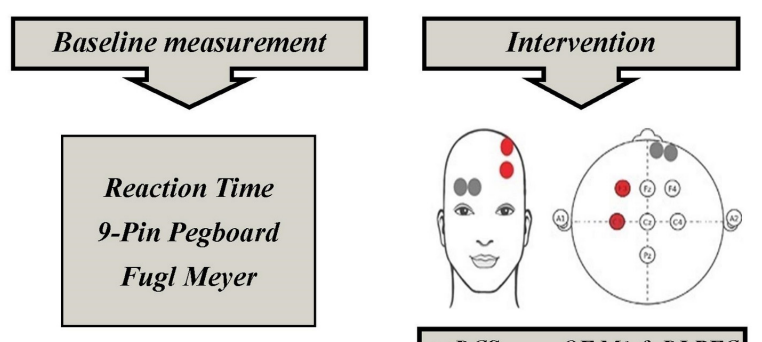

$a-t D C S_{U H C D S} O F M 1 \& D L P F C$
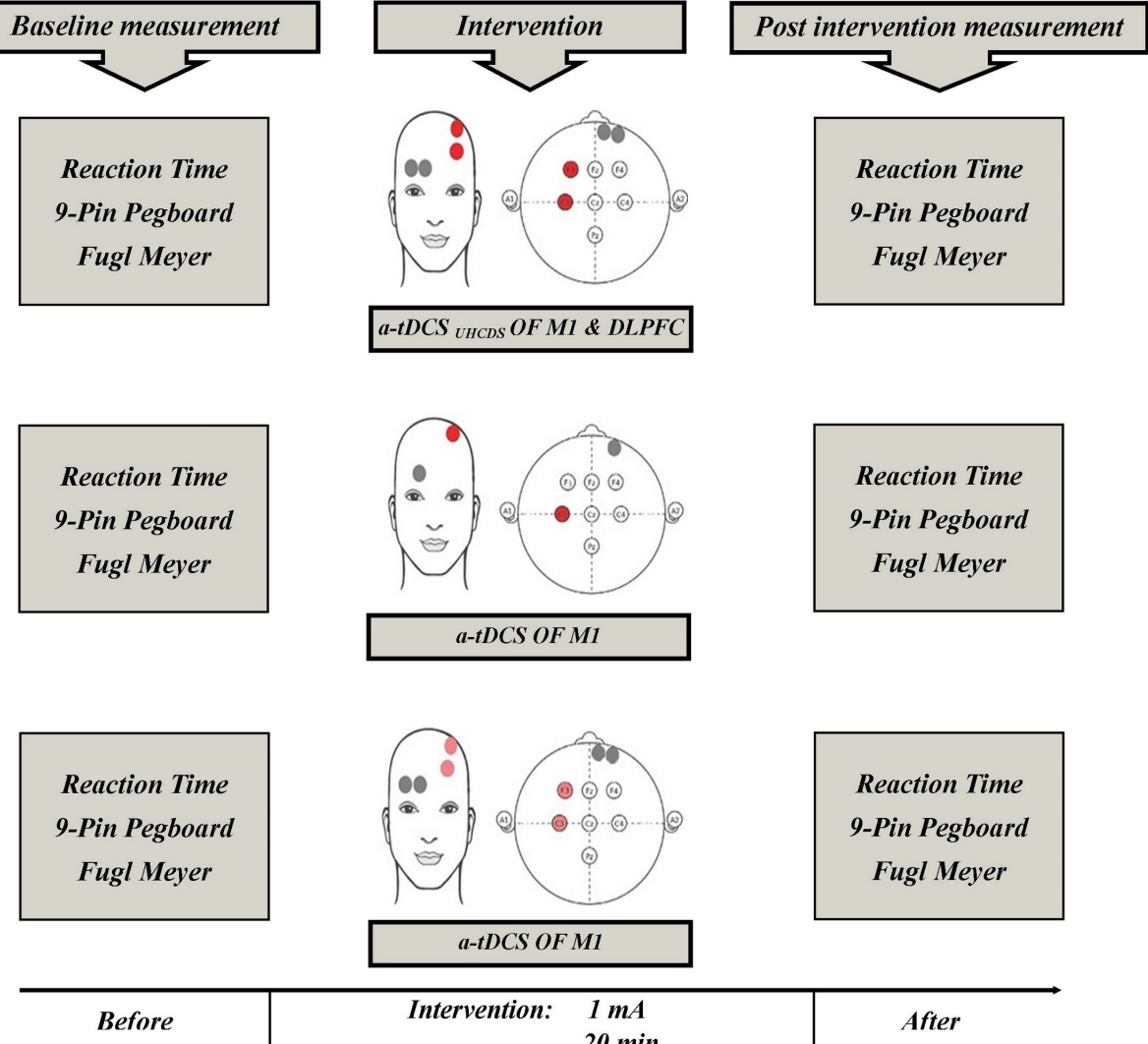

Intervention:

FIGURE 2 | Schematic representation of the experimental protocol with measures taken before and after a-tDCS. a-tDCS, anodal-tDCS; a-tDCS $u H C D S$, anodal unihemispheric concurrent dual-site transcranial direct current stimulation; M1, primary motor cortex; DLPFC, dorsolateral prefrontal cortex.

of Edinburgh, Scotland) was used to assess the hand RT to visual stimuli (Deary et al., 2011). At the start of the test, a number of colored stimuli appeared at random intervals on a laptop screen, which was placed in front of the participant. The participant was asked to press the red slash key on the keyboard as quickly as possible after seeing the stimuli. Participants were in a comfortable position while performing the test; the table height was adjusted such that the person could see the screen and had easy access to the keyboard. Before beginning the study protocol, all participants performed a test trial involving eight stimuli to become familiar with the test. During the study, the program was set to display 20 stimuli. A reduced RT in response to the visual stimuli indicated an improvement in performance. The software has been shown to have good reliability (Deary et al., 2011).

\section{Nine-Pin Pegboard Test}

The 9-PPB test was developed to investigate the hand dexterity in stroke patients (Oxford Grice et al., 2003). The 9-PPB consisted of two rectangular boards that were attached; one board had nine holes in three rows and other board, which was located above the first, had nine holes with a peg in each holes. The 9-PPB was placed in front of each participant who was instructed to pick up each peg from the top board, one by one, as quickly as possible and place it in a hole in the other board. The total time taken to complete the task was determined using a chronometer and was recorded as the test score for each individual (Croarkin et al., 2004). The chronometer accuracy was $0.01 \mathrm{~s}$. The 9-PPB test has excellent test-retest reliability in acute stroke patients (Heller et al., 1987).

\section{Fugl-Meyer Assessment}

The FMA was used to assess upper limb motor function in stroke patients (Fugl-Meyer et al., 1974; Nitsche and Paulus, 2000). The upper limb section of the assessment contained 33 items and each item had the following score: $0=$ unable to move the limb; 1 = partially able to move the limb; and 2 = fully able to move the limb. The total scores for the upper limb section ranged from 0 to 66. Previous studies have reported excellent inter-rater and intra-rater reliability and good construct validity for the FMA (Gladstone et al., 2002).

\section{Procedure}

Figure 2 shows the experimental setup of the study. Participants were asked to sit on an adjustable chair. Participants became familiar with the testing procedures and the outcome measures, which included the FMA, 9-PPB, and RT. Just before and immediately after each experimental intervention, which were given $72 \mathrm{~h}$ apart, the outcome measures were assessed and 
TABLE 2 | Visual Analog Scale Scores for fatigue, attention, and discomfort measurements before and after each intervention.

\begin{tabular}{|c|c|c|c|c|c|c|}
\hline & \multicolumn{2}{|c|}{ Discomfort (Mansur et al., 2005) } & \multicolumn{2}{|c|}{ Attention (Mansur et al., 2005) } & \multicolumn{2}{|c|}{ Fatigue (Mansur et al., 2005) } \\
\hline & After & Before & After & Before & After & Before \\
\hline \multirow[t]{2}{*}{ Sham } & $1.60 \pm 0.91$ & $1.73 \pm 1.03$ & $6.87 \pm 0.35$ & $6.80 \pm 0.41$ & 1.00 & $1.13 \pm 0.35$ \\
\hline & \multicolumn{2}{|c|}{$p=0.164$} & \multicolumn{2}{|c|}{$p=0.334$} & \multicolumn{2}{|c|}{$p=0.164$} \\
\hline \multirow[t]{2}{*}{ a-tDCS } & $1.33 \pm 0.90$ & $1.67 \pm 0.97$ & $6.93 \pm 0.25$ & $6.80 \pm 0.41$ & 1.00 & $1.20 \pm 0.41$ \\
\hline & \multicolumn{2}{|c|}{$p=0.082$} & \multicolumn{2}{|c|}{$p=0.164$} & \multicolumn{2}{|c|}{$p=0.055$} \\
\hline \multirow[t]{2}{*}{ a-tDCS $U H C D S$} & $1.47 \pm 0.91$ & $1.67 \pm 1.13$ & $6.93 \pm 0.25$ & $6.80 \pm 0.56$ & 1.00 & $1.20 \pm 0.41$ \\
\hline & \multicolumn{2}{|c|}{$p=0.082$} & \multicolumn{2}{|c|}{$p=0.164$} & \multicolumn{2}{|c|}{$p=0.082$} \\
\hline
\end{tabular}

Data showing Mean \pm SD of group, scores: 1-7 (1: no attention, 7: full attention; 1: no fatigue, 7: highest fatigue level; 1: no pain, 7: maximum pain).

the participants' scores were measured. Other factors, such as attention, fatigue, and discomfort, were also assessed using a 7 -grade Visual Analog Scale (VAS) to evaluate any adverse or side effects of the tDCS. The participants were asked to show the intensity of their fatigue, attention and discomfort on the $7 \mathrm{~cm}$ VAS just before and immediately after the experimental interventions. The scoring methods for the scales were as follows: $1=$ no concentration and $7=$ maximum concentration; 1 = no fatigue and 7 = maximum fatigue; and $1=$ no discomfort and $7=$ maximum discomfort, respectively. The VAS scoring used in this process has been reported to have excellent reliability (Aitken, 1969; Scherder and Bouma, 2000).

\section{Data Analysis}

Data were analyzed using SPSS version 21 (IBM Corp., Armonk, NY, United States). The normal distribution of the data was assessed using the Shapiro-Wilk test. The effects of the different stimulation conditions on the outcome measures, which included RT, 9-PPB, and FMA, were evaluated using the repeated measures analysis of variance (ANOVA). A comparison of the baseline measurements before each stimulation condition was conducted using the one-way ANOVA. The Wilcoxon test was used to compare the psychological data before and after each stimulation condition. The level of significance was set at $p<0.05$.

TABLE 3 | Mean and SD of RT, 9-PPT, and FMA in three stimulation conditions.

\begin{tabular}{lccc}
\hline & \multicolumn{1}{c}{ Sham } & a-tDcs & a-tDcs \\
\hline RT $(s)$ & & & \\
Pre & $0.670 \pm 0.048$ & $0.604 \pm 0.054$ & $0.625 \pm 0.052$ \\
Post & $0.665 \pm 0.048 .91$ & $0.607 \pm 0.048$ & $0.577 \pm 0.037$ \\
9-PPT (s) & & & \\
Pre & $70.76 \pm 5.56$ & $74.33 \pm 6.40$ & $70.23 \pm 6.13$ \\
Post & $70.38 \pm 5.96$ & $71.09 \pm 5.80$ & $65.75 \pm 5.47$ \\
FMA & $38.20 \pm 1.47$ & & \\
Pre & $38.46 \pm 1.50$ & $38.26 \pm 1.46$ & $38.20 \pm 1.43$ \\
Post & $9-P P B=1.46$ & $38.53 \pm 1.41$ \\
\hline
\end{tabular}

$R T$, reaction time; 9-PPB, nine-pin pegboard; FMA, Fugl-Meyer assessment;

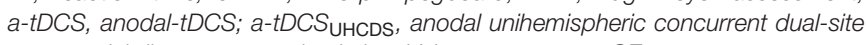
transcranial direct current stimulation. Values are mean $\pm S E$.

\section{RESULTS}

The analysis showed that the distribution of the psychological data was not normal. However, the RT, 9-PPB, and FMA data were distributed normally.

\section{Psychological Data}

An analysis of the psychological data revealed that there was no significant difference between the pre- and post-procedure attention, fatigue, and discomfort of the participants. No participant reported a headache or any other adverse effect after receiving the tDCS; however, two participants had the feeling of burning at the point of the active electrode at the start of the stimulation, which was resolved after $1 \mathrm{~min}$. Table 2 shows patients' perceived VAS scores in centimeter for the assessed psychological variables including fatigue, attention, and discomfort just before and immediately after each experimental condition.

\section{Comparison of Baseline Values}

The one-way ANOVA showed that there were no significant difference between the baseline RT $\left[F_{(2,28)}=2.56, p>0.05\right]$, 9-PPB $[F(2,28)=1.638, p>0.05]$ and FMA $\left[F_{(2,28)}=0.318\right.$, $p>0.05]$ for the sham stimulation, a-tDCS, and a-tDCS $\mathrm{tHCDS}_{\text {: }}$ (Table 3). Thus, the baseline for the sham stimulation was used as baseline measurement for all conditions in data analyses.

\section{Reaction Time}

The repeated measures ANOVA showed the type of stimulation had a significant effect on the RT: $F_{(2.087,29.224)}=4.96, p<0.05$ (Figure 3). The Bonferroni correction showed that after applying a-tDCS ${ }_{U H C D S}$, there was a significant lower RT compared with the sham stimulation $(p=0.031)$. However, there was no significant difference between a-tDCS and a-tDCS ${ }_{U H C D S}$ $(p>0.05)$.

\section{Nine-Pin Pegboard Test}

The repeated measures ANOVA showed the type of stimulation had a significant effect on the completion time for the 9-PPB: $F_{(3,42)}=5.997, p<0.05$ (Figure 4). There was a significant lower completion time after applying a-tDCS ${ }_{U H C D S}$ compared with the sham stimulation $(p=0.036)$ and a-tDCS $(p=0.015)$. However, there was no significant difference between a-tDCS and the sham stimulation $(p>0.05)$. 


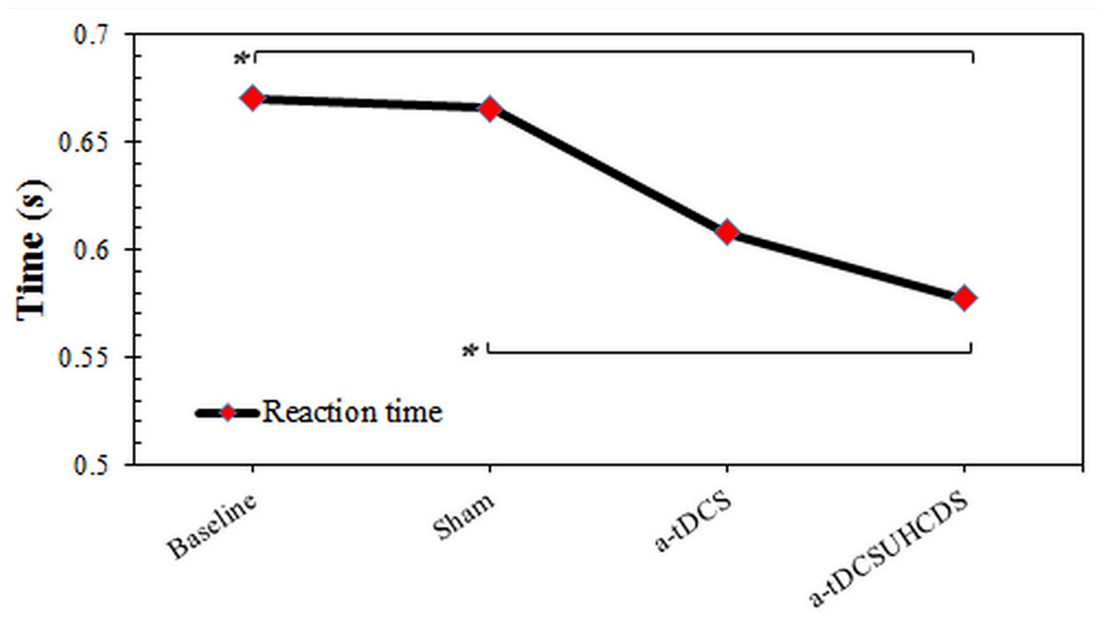

FIGURE 3 | The comparison of reaction time (mean $\pm \mathrm{SD}$ ) before and after the stimulation. a-tDCS, anodal-tDCS; a-tDCS $U H C D S$, anodal unihemispheric concurrent dual-site transcranial direct current stimulation; *, significant.

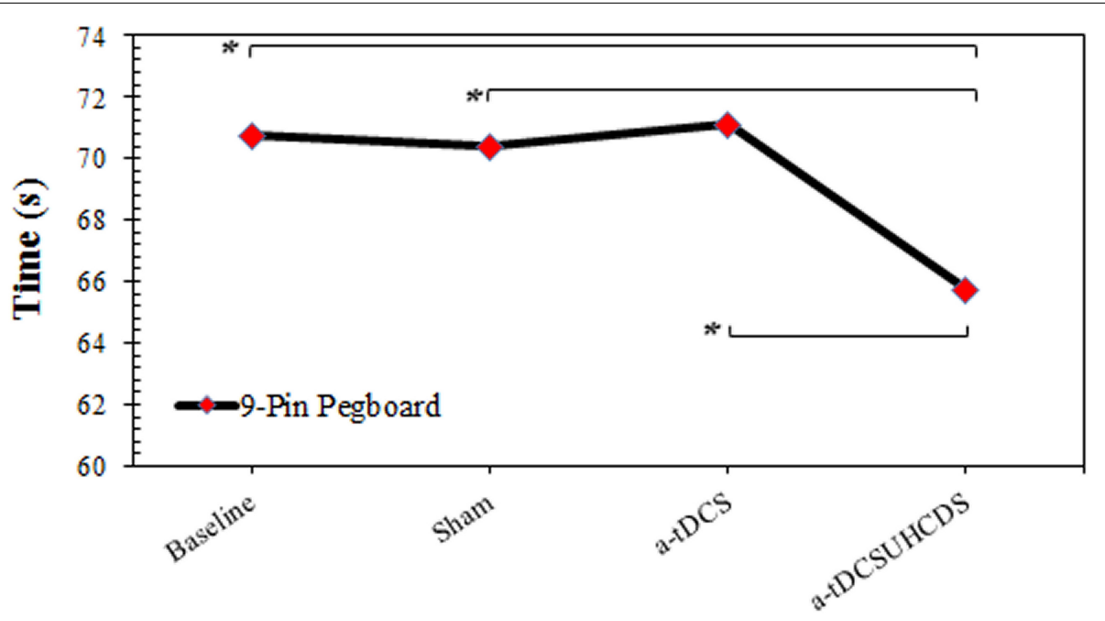

FIGURE 4 | The comparison of completion time of the 9-pin pegboard test (mean \pm SD) before and after the stimulation. a-tDCS, anodal-tDCS; a-tDCS $4 H C D S$, anodal unihemispheric concurrent dual-site transcranial direct current stimulation; *, significant.

\section{Fugl-Meyer Assessment}

Comparing baseline values and post-stimulation values showed the effect of the type of stimulation on the FMA was not significant. The repeated measures ANOVA showed that the stimulation had no significant effect on the FMA scores: $F_{(3,42)}=2.364, p>0.05$ (Figure 5).

\section{DISCUSSION}

\section{Baseline Measurements}

There were no significant differences between the baseline measurements of the dependent variables (i.e., RT, FMA, and 9-PPB) for the three experimental conditions. This similarity indicated that the length of the wash-out period was enough to avoid the carry-over effect between the stimulation conditions.

\section{Safety and Side Effects of a-tDCS $S_{U H C D S}$}

Fatigue, attention, and discomfort can influence motor performance. However, there was no significant difference between the pre- and post-procedure for these scales in this study, so we concluded fatigue, attention and discomfort did not affect out the results.

\section{Effect of a-tDCS ${ }_{U H C D S}$ on Reaction Time}

The results of this study indicated that a-tDCS ${ }_{U H C D S}$ had a more significant effect on the reduction of RT than the single-site a-tDCS and the sham stimulation. The findings supported the hypothesis that a-tDCS $S_{U H C D S}$ of the M1-DLPFC would induce a larger decrease in RT than single-site a-tDCS of the M1. This result agreed with the findings of Vaseghi et al. (2015b) who reported that the simultaneous stimulation of the M1 and DLPFC increased the CSE in healthy individuals. There is a functional connectivity between the different brain regions (Keeser et al., 


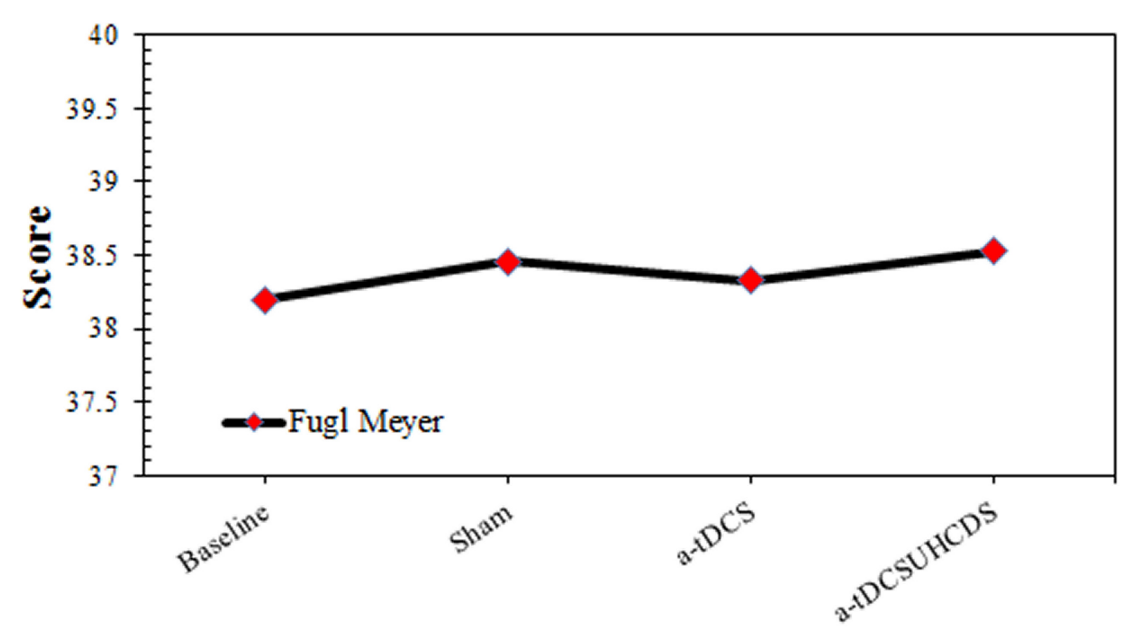

FIGURE 5 | The comparison of Fugl-Meyer assessment (mean \pm SD) before and after the stimulation. a-tDCS, anodal-tDCS; a-tDCS $u H C D S$, anodal unihemispheric concurrent dual-site transcranial direct current stimulation.

2011; Luft et al., 2014). It has been shown that the premotor cortex consists of dorsal and ventral sections. The outputs of the dorsal section are sent to the M1 and spinal cord, while the premotor cortex receives inputs from the DLPFC (Dum and Strick, 1991; He et al., 1993). Therefore, we can conclude that the M1 and DLPFC are indirectly connected. Studies have also shown the functional connectivity between M1 and DLPFC (Vaseghi et al., 2015b, 2016). Therefore, compared with single-site a-tDCS of the M1, a-tDCS of the M1-DLPFC activates the DLPFCpremotor cortex-M1 pathway (Bunge et al., 2001; Nitsche and Paulus, 2001; Van Ryckeghem et al., 2013), which may have a profound effect on cortical and behavioral outcome measures, such as motor RT. In addition, there is also a connection between the ventral premotor section and the prefrontal cortex (Hutchins et al., 1988; Lu et al., 1994). Therefore, stimulating the prefrontal cortex can influence the ventral premotor section, which contains the upper limb representations (Hutchins et al., 1988; Lu et al., 1994). It should be noted that any improvement in performance, such as a reduced RT, requires high levels of cognition and improved motor performance (Salthouse, 1996; Madden, 2001). Cappon et al. (2016) in a comprehensive systematic review reported that a-tDCS stimulation of DLPFC could improve cognitive impairments in chronic stroke patients. Therefore, the simultaneous stimulation of the M1 and DLPFC not only favorably affects the CSE, but also improves the performance by decreasing the RT (Salthouse, 1996). In other words, an improved RT requires both motor and cognitive functions and the simultaneous stimulation of the M1 and DLPFC may cause motor and cognitive improvement.

Hummel et al. (2006) showed that one session of a-tDCS of M1 could significantly reduce the RT. This finding disagreed with the results of the current study because we did not observe any significant changes in the RTs of patients' upper limbs after a-tDCS of the M1. The discrepancy between the results may be explained by the fact that different assessment tools were used. In the present study, the clinical measurements of RT were used, while Hummel et al. (2006) used electromyography to assess the RT.

\section{Effect of a-tDCS $U$ UHCDS on Nine-Pin Pegboard Test}

The results of the present study indicated that there was a significant reduction in the completion time of the 9-PPB test after a-tDCS $S_{U H C D S}$ compared with the single-site a-tDCS and the sham stimulation. This finding supported our hypothesis that a simultaneous stimulation of ipsilateral M1 and DLPFC would improve the 9-PPB completion time. This finding was consistent with the study by Vaseghi et al. (2015b), which showed an improvement in M1 excitability and motor function following the dual-site stimulation of the M1 and DLPFC. It is believed that the 9-PPB test involves a neural network for transferring the touch and visual information to the opposite M1 of the involved hand. Therefore, the 9-PPB test involves the integration of sensory, motor, and cognitive processes (Talati et al., 2005). Other studies have claimed that a-tDCS of the DLPFC improves performance in several cognitive domains, including executive functions (Keeser et al., 2011); thus, the present findings seemed reasonable. In addition, there are a number of well-known projections between the DLPFC, the cingulate cortex, and the parietal lobe. Therefore, it might be assumed that a-tDCS of the DLPFC increases the interaction between these pathways, which form part of the so-called "attention" network. Increased connectivity within this network was also reported following cognitive training and application of a-tDCS. Therefore, it is quite possible that the simultaneous stimulation of the M1 and DLPFC improves a patient's attention, which consequently leads to a better performance in completing the 9-PPB test (Fransson, 2005; van de Ven et al., 2008; Keeser et al., 2011). In addition, Bachtiar et al. (2015) reported that a-tDCS of the M1 causes an increase in CSE. This increase coincided with a reduction in the GABAergic inhibitory system (Ziemann et al., 1998; Garry et al., 2004; Vaseghi et al., 2015b), as evidenced by a decrease in 
the short interval intracortical inhibition (Nitsche et al., 2005). Increased CSE in the involved cerebral hemispheres may help in the recovery of motor function of affected limbs after a stroke, which may lead to a better performance during the 9-PPB test.

\section{Effect of a-tDCS UHCDS $_{\text {on Fugl-Meyer }}$ Assessment}

The results of the present study showed that there was no significant difference between the scores of FMA for the different experimental conditions. This finding partly agreed with a previous studies that found there was no improvement in upper limb performance following a-tDCS of the M1 (Hesse et al., 2007; Rossi et al., 2013). In the majority of studies, a-tDCS was used as a priming technique concurrently with functional training or robotic upper limb therapy. While some of these studies reported an improvement in the FMA, no difference was observed between the a-tDCS and control groups (Kim et al., 2010; Cha et al., 2014). Hesse et al. (2011) assessed the priming effects of a-tDCS with and without robotic-assisted arm training on the motor function in subacute stroke patients. Both study groups showed an improvement in the FMA, although there was no significant difference between the two experimental conditions (Hesse et al., 2011).

Rocha et al. (2016) evaluated the effect of a-tDCS on upper limb function in chronic stroke survivors. They reported an improvement in FMA scores after a-tDCS compared with the sham stimulation. This finding did not agree with the findings in the current study. This discrepancy could be explained by the methodological differences between the studies. In the Rocha et al. (2016) study, patients received 12 a-tDCS sessions, while patients in the present study only experienced a single a-tDCS session. It may be concluded that one session of intervention was insufficient to induce an improvement in the motor function of the upper limbs (Triccas et al., 2015; Rocha et al., 2016). In addition, the sample size calculation for the present study was performed based on time-based variables such as RT and 9-PPB and not based on function-based variables like FMA. Therefore, it is possible that the small sample size of the present study resulted in insignificant finding for FMA.

\section{Limitations and Suggestions for Future Research}

There are some limitations of the present study that may affect the interpretation of the results. First, the target population was subacute stroke patients. Therefore, it might not be possible to generalize the results to chronic stroke survivors. As it is believed that the neuroplasticity of the nervous system changes over time, the effects of a-tDCS may be different in chronic stroke patients. The second limitation is that we did not directly examine the cortex excitability. Future studies should evaluate the excitability of the cortex directly using a-tDCS $S_{U H C D S}$. Despite these limitations, this is the first study that used the novel tDCS on stroke survivors.

A future study with more a-tDCS ${ }_{U H C D S}$ treatment sessions, especially in combination with other rehabilitation programs, will shed more light on the rehabilitation of upper limb motor function in stroke survivors. Vines et al. (2008) suggested that the addition of cathodal tDCS (c-tDCS) on the contralateral M1 simultaneously with a-tDCS on the M1 might have a greater effect on motor function than anodal stimulation alone. This may occur because of the balancing act of the cathodal stimulation of the opposite M1, which reduces the inhibitory effects of the intact brain on the involved side (Bradnam et al., 2011). Future studies are recommended to evaluate the effects of this balancing act through the cathodal stimulation of the uninvolved hemisphere. Finally, the small sample size in the present study might limit us to give a robust conclusion especially on insignificant findings and compare and investigate effects of the site of lesion on motor improvement. Future studies with larger sample size and comparable healthy group are recommended. ${ }^{1}$

\section{CONCLUSION}

The present study investigated the difference between the effects of conventional single-site stimulation of M1 and unihemispheric concurrent dual-site a-tDCS of the M1 and DLPFC on upper limb motor function of subacute stroke patients. We concluded that the simultaneous stimulation of the M1 and DLPFC induced a more significant reduction in RT and completion time for the 9PPB test and, thus, caused more improvement in hand function.

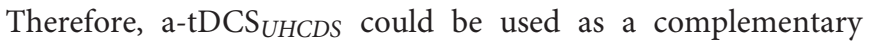
treatment for the improvement of upper limb motor function in stroke patients.

\section{DATA ACCESSIBILITY}

The data are accessible from the authors. They are not uploaded into public repositories, because the ethics agreement did not contain this.

\section{AUTHOR CONTRIBUTIONS}

LR, NK, IA, and SJ designed the experiments. STA collected the data. LR, STA, NK, IA, SJ, and SAA analyzed and interpreted the data and wrote the manuscript.

\section{ACKNOWLEDGMENTS}

We wish to thank the participants and health care professionals for their support of this study. We would also like to thank the University of Social Welfare and Rehabilitation Sciences, the Iranian Red Crescent Society, and Rofeideh Rehabilitation Hospital for helping us recruit patients for this study.

\section{SUPPLEMENTARY MATERIAL}

The Supplementary Material for this article can be found online at: https://www.frontiersin.org/articles/10.3389/fnhum. 2018.00441/full\#supplementary-material

\footnotetext{
${ }^{1}$ http://www.irct.ir
} 


\section{REFERENCES}

Aitken, R. C. (1969). Measurement of feelings using visual analogue scales. Proc. $R$. Soc. Med. 62, 989-993.

Andrade, S. M., Batista, L. M., Nogueira, L. L., Oliveira, E. A. D., de Carvalho, A. G., Lima, S. S., et al. (2017). Constraint-induced movement therapy combined with transcranial direct current stimulation over premotor cortex improves motor function in severe stroke: a pilot randomized controlled trial. Rehabil. Res. Pract. 2017:6842549. doi: 10.1155/2017/6842549

Bachtiar, V., Near, J., Johansen-Berg, H., and Stagg, C. J. (2015). Modulation of GABA and resting state functional connectivity by transcranial direct current stimulation. eLife 4:e08789. doi: 10.7554/eLife.08789

Bastani, A., and Jaberzadeh, S. (2012). Does anodal transcranial direct current stimulation enhance excitability of the motor cortex and motor function in healthy individuals and subjects with stroke: a systematic review and metaanalysis. Clin. Neurophysiol. 123, 644-657. doi: 10.1016/j.clinph.2011.08.029

Bastani, A., and Jaberzadeh, S. (2013). a-tDCS differential modulation of corticospinal excitability: the effects of electrode size. Brain Stimul. 6, 932-937. doi: 10.1016/j.brs.2013.04.005

Boggio, P. S., Castro, L. O., Savagim, E. A., Braite, R., Cruz, V. C., Rocha, R. R., et al. (2006). Enhancement of non-dominant hand motor function by anodal transcranial direct current stimulation. Neurosci. Lett. 404, 232-236. doi: 10. 1016/j.neulet.2006.05.051

Bradnam, L. V., Stinear, C. M., Barber, P. A., and Byblow, W. D. (2011). Contralesional hemisphere control of the proximal paretic upper limb following stroke. Cereb. Cortex 22, 2662-2671. doi: 10.1093/cercor/bhr344

Bunge, S. A., Ochsner, K. N., Desmond, J. E., Glover, G. H., and Gabrieli, J. D. (2001). Prefrontal regions involved in keeping information in and out of mind. Brain 124, 2074-2086. doi: 10.1093/brain/124.10.2074

Butler, A. J., Shuster, M., O'hara, E., Hurley, K., Middlebrooks, D., and Guilkey, K. (2013). A meta-analysis of the efficacy of anodal transcranial direct current stimulation for upper limb motor recovery in stroke survivors. J. Hand Ther. 26, 162-171. doi: 10.1016/j.jht.2012.07.002

Cappon, D., Jahanshahi, M., and Bisiacchi, P. (2016). Value and efficacy of transcranial direct current stimulation in the cognitive rehabilitation: a critical review since 2000. Front. Neurosci. 10:157. doi: 10.3389/fnins.2016. 00157

Cha, H.-K., Ji, S.-G., Kim, M.-K., and Chang, J.-S. (2014). Effect of Transcranial Direct Current Stimulation of Function in Patients with Stroke. Journal of physical therapy science 26, 363. doi: 10.1589/jpts.26.363

Croarkin, E., Danoff, J., and Barnes, C. (2004). Evidence-based rating of upperextremity motor function tests used for people following a stroke. Phys. Ther. $84,62-74$.

Deary, I. J., Liewald, D., and Nissan, J. (2011). A free, easy-to-use, computer-based simple and four-choice reaction time programme: the Deary-Liewald reaction time task. Behav. Res. Methods 43, 258-268. doi: 10.3758/s13428-010-0024- 1

Di Lazzaro, V., Dileone, M., Capone, F., Pellegrino, G., Ranieri, F., Musumeci, G., et al. (2014). Immediate and late modulation of interhemipheric imbalance with bilateral transcranial direct current stimulation in acute stroke. Brain stimul. 7, 841-848. doi: 10.1016/j.brs.2014.10.001

Dum, R. P., and Strick, P. L. (1991). The origin of corticospinal projections from the premotor areas in the frontal lobe. J. Neurosci. 11, 667-689. doi: 10.1523/ JNEUROSCI.11-03-00667.1991

Fleming, M. K., Rothwell, J. C., Sztriha, L., Teo, J. T., and Newham, D. J. (2017). The effect of transcranial direct current stimulation on motor sequence learning and upper limb function after stroke. Clin. Neurophysiol. 128, 1389-1398. doi: 10.1016/j.clinph.2017.03.036

Fransson, P. (2005). Spontaneous low-frequency BOLD signal fluctuations: an fMRI investigation of the resting-state default mode of brain function hypothesis. Hum. Brain Mapp. 26, 15-29. doi: 10.1002/hbm.20113

Fugl-Meyer, A. R., Jääskö, L., Leyman, I., Olsson, S., and Steglind, S. (1974). The post-stroke hemiplegic patient. 1. a method for evaluation of physical performance. Scand. J. Rehabil. Med. 7, 13-31.

Fujimoto, S., Kon, N., Otaka, Y., Yamaguchi, T., Nakayama, T., Kondo, K., et al. (2016). Transcranial direct current stimulation over the primary and secondary somatosensory cortices transiently improves tactile spatial discrimination in stroke patients. Front. Neurosci. 10:128. doi: 10.3389/fnins.2016. 00128
Furubayashi, T., Terao, Y., Arai, N., Okabe, S., Mochizuki, H., Hanajima, R., et al. (2008). Short and long duration transcranial direct current stimulation (tDCS) over the human hand motor area. Exp. Brain Res. 185, 279-286. doi: 10.1007/ s00221-007-1149-z

Garry, M. I., Kamen, G., and Nordstrom, M. A. (2004). Hemispheric differences in the relationship between corticomotor excitability changes following a finemotor task and motor learning. J. Neurophysiol. 91, 1570-1578. doi: 10.1152/jn. 00595.2003

Gladstone, D. J., Danells, C. J., and Black, S. E. (2002). The Fugl-Meyer assessment of motor recovery after stroke: a critical review of its measurement properties. Neurorehabil. Neural Repair 16, 232-240. doi: 10.1177/154596802401105171

Harris, J. E., and Eng, J. J. (2007). Paretic upper-limb strength best explains arm activity in people with stroke. Phys. Ther. 87, 88-97. doi: 10.2522/ptj.2006 0065

He, S.-Q., Dum, R. P., and Strick, P. L. (1993). Topographic organization of corticospinal projections from the frontal lobe: motor areas on the lateral surface of the hemisphere. J. Neurosci. 13, 952-980. doi: 10.1523/JNEUROSCI. 13-03-00952.1993

Heller, A., Wade, D., Wood, V. A., Sunderland, A., Hewer, R. L., and Ward, E. (1987). Arm function after stroke: measurement and recovery over the first three months. J. Neurol. Neurosurg. Psychiatry 50, 714-719. doi: 10.1136/jnnp. 50.6.714

Hesse, S., Waldner, A., Mehrholz, J., Tomelleri, C., Pohl, M., and Werner, C. (2011). Combined transcranial direct current stimulation and robot-assisted arm training in subacute stroke patients an exploratory, randomized multicenter trial. Neurorehabil. Neural Repair 25, 838-846. doi: 10.1177/15459683114 13906

Hesse, S., Werner, C., Schonhardt, E., Bardeleben, A., Jenrich, W., and Kirker, S. (2007). Combined transcranial direct current stimulation and robot-assisted arm training in subacute stroke patients: a pilot study. Restor. Neurol. Neurosci. $25,9-15$.

Hummel, F., Celnik, P., Giraux, P., Floel, A., Wu, W.-H., Gerloff, C., et al. (2005). Effects of non-invasive cortical stimulation on skilled motor function in chronic stroke. Brain 128, 490-499. doi: 10.1093/brain/awh369

Hummel, F., and Cohen, L. G. (2005). Improvement of motor function with noninvasive cortical stimulation in a patient with chronic stroke. Neurorehabil. Neural Repair 19, 14-19. doi: 10.1177/1545968304272698

Hummel, F. C., and Cohen, L. G. (2006). Non-invasive brain stimulation: a new strategy to improve neurorehabilitation after stroke? Lancet Neurol. 5, 708-712. doi: 10.1016/S1474-4422(06)70525-7

Hummel, F. C., Voller, B., Celnik, P., Floel, A., Giraux, P., Gerloff, C., et al. (2006). Effects of brain polarization on reaction times and pinch force in chronic stroke. BMC Neurosci. 7:73. doi: 10.1186/1471-2202-7-73

Hutchins, K., Martino, A., and Strick, P. (1988). Corticospinal projections from the medial wall of the hemisphere. Exp. Brain Res. 71, 667-672. doi: 10.1007/ BF00248761

Karok, S., and Witney, A. G. (2013). Enhanced motor learning following taskconcurrent dual transcranial direct current stimulation. PLoS One 8:e85693. doi: 10.1371/journal.pone.0085693

Keeser, D., Meindl, T., Bor, J., Palm, U., Pogarell, O., Mulert, C., et al. (2011). Prefrontal transcranial direct current stimulation changes connectivity of resting-state networks during fMRI. J. Neurosci. 31, 15284-15293. doi: 10.1523/ JNEUROSCI.0542-11.2011

Kim, D.-Y., Lim, J.-Y., Kang, E. K., You, D. S., Oh, M.-K., Oh, B.-M., et al. (2010). Effect of transcranial direct current stimulation on motor recovery in patients with subacute stroke. Am. J. Phys. Med. Rehabil. 89, 879-886. doi: 10.1097/ PHM.0b013e3181f70aa7

Kim, K.-U., Kim, S.-H., and An, T.-G. (2016). Effect of transcranial direct current stimulation on visual perception function and performance capability of activities of daily living in stroke patients. J. Phys. Ther. Sci. 28, 2572-2575. doi: $10.1589 /$ jpts.28.2572

Kim, K.-Y., Sim, K.-C., Kim, H.-S., Choi, W.-S., and Kim, G.-D. (2012). Analgesic effects of transcranial direct current stimulation on central neuropathic pain in spinal cord contusive rat model. Int. J. Contents 8, 74-81. doi: 10.5392/IJoC. 2012.8.1.074

Kuklina, E. V., Tong, X., George, M. G., and Bansil, P. (2012). Epidemiology and prevention of stroke: a worldwide perspective. Expert Rev. Neurother. 12, 199-208. doi: 10.1586/ern.11.99 
Kwon, Y. H., Ko, M.-H., Ahn, S. H., Kim, Y.-H., Song, J. C., Lee, C.-H., et al. (2008). Primary motor cortex activation by transcranial direct current stimulation in the human brain. Neurosci. Lett. 435, 56-59. doi: 10.1016/j.neulet.2008.02.012

Lee, D.-G., and Lee, D.-Y. (2015). Effects of adjustment of transcranial direct current stimulation on motor function of the upper extremity in stroke patients. J. Phys. Ther. Sci. 27, 3511-3513. doi: 10.1589/jpts.27.3511

Liebetanz, D., Nitsche, M. A., Tergau, F., and Paulus, W. (2002). Pharmacological approach to the mechanisms of transcranial DC-stimulation-induced aftereffects of human motor cortex excitability. Brain 125, 2238-2247. doi: 10.1093/ brain/awf238

Lu, M. T., Preston, J. B., and Strick, P. L. (1994). Interconnections between the prefrontal cortex and the premotor areas in the frontal lobe. J. Comp. Neurol. 341, 375-392. doi: 10.1002/cne.903410308

Luft, C. D. B., Pereda, E., Banissy, M. J., and Bhattacharya, J. (2014). Best of both worlds: promise of combining brain stimulation and brain connectome. Front. Syst. Neurosci. 8:132. doi: 10.3389/fnsys.2014.00132

Madden, D. J. (2001). Speed and timing of behavioral processes. Handb. Psychol. Aging 5, 288-312.

Mansur, C., Fregni, F., Boggio, P., Riberto, M., Gallucci-Neto, J., Santos, C., et al. (2005). A sham stimulation-controlled trial of rTMS of the unaffected hemisphere in stroke patients. Neurology 64, 1802-1804. doi: 10.1212/01.WNL. 0000161839.38079 .92

Mayo, N. E., Wood-Dauphinee, S., Ahmed, S., Gordon, C., Higgins, J., McEwen, S., et al. (1999). Disablement following stroke. Disabil. Rehabil. 21, 258-268. doi: $10.1080 / 096382899297684$

Nitsche, M., and Paulus, W. (2000). Excitability changes induced in the human motor cortex by weak transcranial direct current stimulation. J. Physiol. 527, 633-639. doi: 10.1111/j.1469-7793.2000.t01-1-00633.x

Nitsche, M. A., Doemkes, S., Karakoese, T., Antal, A., Liebetanz, D., Lang, N., et al. (2007). Shaping the effects of transcranial direct current stimulation of the human motor cortex. J. Neurophysiol. 97, 3109-3117. doi: 10.1152/jn.01312. 2006

Nitsche, M. A., and Paulus, W. (2001). Sustained excitability elevations induced by transcranial DC motor cortex stimulation in humans. Neurology 57, 1899-1901. doi: 10.1212/WNL.57.10.1899

Nitsche, M. A., Seeber, A., Frommann, K., Klein, C. C., Rochford, C., Nitsche, M. S., et al. (2005). Modulating parameters of excitability during and after transcranial direct current stimulation of the human motor cortex. J. Physiol. 568, 291-303. doi: 10.1113/jphysiol.2005.092429

Oxford Grice, K., Vogel, K. A., Le, V., Mitchell, A., Muniz, S., and Vollmer, M. A. (2003). Adult norms for a commercially available Nine Hole Peg Test for finger dexterity. Am. J. Occup. Ther. 57, 570-573. doi: 10.5014/ajot.57.5.570

Parker, V., Wade, D., and Hewer, R. L. (1986). Loss of arm function after stroke: measurement, frequency, and recovery. Disabil. Rehabil. 8, 69-73. doi: 10.3109/ 03790798609166178

Rocha, S., Silva, E., Foerster, Á., Wiesiolek, C., Chagas, A. P., Machado, G., et al. (2016). The impact of transcranial direct current stimulation (tDCS) combined with modified constraint-induced movement therapy (mCIMT) on upper limb function in chronic stroke: a double-blind randomized controlled trial. Disabil. Rehabil. 38, 653-660. doi: 10.3109/09638288.2015.1055382

Rossi, C., Sallustio, F., Di Legge, S., Stanzione, P., and Koch, G. (2013). Transcranial direct current stimulation of the affected hemisphere does not accelerate recovery of acute stroke patients. Eur. J. Neurol. 20, 202-204. doi: 10.1111/j. 1468-1331.2012.03703.x

Salthouse, T. A. (1996). The processing-speed theory of adult age differences in cognition. Psychol. Rev. 103:403. doi: 10.1037/0033-295X.103.3.403

Satow, T., Kawase, T., Kitamura, A., Kajitani, Y., Yamaguchi, T., Tanabe, N., et al. (2016). Combination of transcranial direct current stimulation and neuromuscular electrical stimulation improves gait ability in a patient in chronic stage of stroke. Case Rep. Neurol. 8, 39-46. doi: 10.1159/000444167

Scherder, E. J., and Bouma, A. (2000). Visual analogue scales for pain assessment in Alzheimer's disease. Gerontology 46, 47-53. doi: 10.1159/000022133

Stagg, C. J., Lin, R. L., Mezue, M., Segerdahl, A., Kong, Y., and Xie, J. (2013). Widespread modulation of cerebral perfusion induced during and after transcranial direct current stimulation applied to the left dorsolateral prefrontal cortex. J. Neurosci. 33, 11425-11431. doi: 10.1523/JNEUROSCI.3887-12.2013

Summers, J. J., Kagerer, F. A., Garry, M. I., Hiraga, C. Y., Loftus, A., and Cauraugh, J. H. (2007). Bilateral and unilateral movement training on upper limb function in chronic stroke patients: a TMS study. J. Neurol. Sci. 252, 76-82. doi: 10.1016/ j.jns.2006.10.011

Talati, A., Valero-Cuevas, F. J., and Hirsch, J. (2005). Visual and tactile guidance of dexterous manipulation tasks: an fMRI study. Percept. Mot. Skills 101, 317-334. doi: 10.2466/pms.101.1.317-334

Triccas, L. T., Burridge, J. H., Hughes, A., Verheyden, G., Desikan, M., and Rothwell, J. (2015). A double-blinded randomised controlled trial exploring the effect of anodal transcranial direct current stimulation and uni-lateral robot therapy for the impaired upper limb in sub-acute and chronic stroke. NeuroRehabilitation 37, 181-191. doi: 10.3233/NRE-151251

van de Ven, V., Bledowski, C., Prvulovic, D., Goebel, R., Formisano, E., Di Salle, F., et al. (2008). Visual target modulation of functional connectivity networks revealed by self-organizing group ICA. Hum. Brain Mapp. 29, 1450-1461. doi: 10.1002/hbm.20479

Van Ryckeghem, D. M., De Houwer, J., Van Bockstaele, B., Van Damme, S., De Schryver, M., and Crombez, G. (2013). Implicit associations between pain and self-schema in patients with chronic pain. Pain 154, 2700-2706. doi: 10.1016/j. pain.2013.07.055

Vaseghi, B., Zoghi, M., and Jaberzadeh, S. (2015a). How does anodal transcranial direct current stimulation of the pain neuromatrix affect brain excitability and pain perception? A randomised, double-blind, sham-control study. PLoS One 10:e0118340. doi: 10.1371/journal.pone.0118340

Vaseghi, B., Zoghi, M., and Jaberzadeh, S. (2015b). The effects of anodal-tDCS on corticospinal excitability enhancement and its after-effects: conventional vs. unihemispheric concurrent dual-site stimulation. Front. Hum. Neurosci. 9:533. doi: 10.3389/fnhum.2015.00533

Vaseghi, B., Zoghi, M., and Jaberzadeh, S. (2016). Unihemispheric concurrent dualsite cathodal transcranial direct current stimulation: the effects on corticospinal excitability. Eur. J. Neurosci. 43, 1161-1172. doi: 10.1111/ejn.13229

Vines, B. W., Cerruti, C., and Schlaug, G. (2008). Dual-hemisphere tDCS facilitates greater improvements for healthy subjects' non-dominant hand compared to uni-hemisphere stimulation. BMC Neurosci. 9:103. doi: 10.1186/1471-2202-9103

Ziemann, U., Hallett, M., and Cohen, L. G. (1998). Mechanisms of deafferentationinduced plasticity in human motor cortex. J. Neurosci. 18, 7000-7007. doi: 10.1523/JNEUROSCI.18-17-07000.1998

Conflict of Interest Statement: The authors declare that the research was conducted in the absence of any commercial or financial relationships that could be construed as a potential conflict of interest.

Copyright (C) 2018 Achacheluee, Rahnama, Karimi, Abdollahi, Arslan and Jaberzadeh. This is an open-access article distributed under the terms of the Creative Commons Attribution License (CC BY). The use, distribution or reproduction in other forums is permitted, provided the original author(s) and the copyright owner(s) are credited and that the original publication in this journal is cited, in accordance with accepted academic practice. No use, distribution or reproduction is permitted which does not comply with these terms. 